\title{
嵌埋于极化 $\mathrm{BaTiO}_{3}$ 薄膜内的 $\mathrm{Ag}$ 纳米微粒 对基体中二次谐波产生的增强作用
}

近年来, 铁电薄膜的光学性质引起了许 多研究者的注意. 除较为常用的电光效应 外, 人们还发现了极化后的 $\mathrm{BaTiO}_{3}$ 薄膜在激 光辐射下产生很强的二次谐波 ${ }^{[1]}$, 表明这类 材料在非线性光学领域有很好的应用前景. 最近, 我们利用 sol-gel 过程制备具有嵌埋结 构的铁电薄膜一一金属纳米微粒体系, 发现 这类新型材料具有很特殊的光吸收性质 ${ }^{[2]}$. 本文对这类材料的二次非线性光学性质进行 了研究, 发现金属纳米微粒对铁电薄膜中二 次谐波的产生有明显的增强作用.

实验所用的样品为淀积在石英玻璃基片 上的、解埋 $\mathrm{Ag}$ 纳米微粒的 $\mathrm{BaTiO}_{3}$ 薄膜, 用 sol-gel 法制备, 具体过程见文献 [2]. 石英基 片的厚度为 $1 \mathrm{~mm}$, 面积约为 $1.5 \mathrm{~cm}^{2}$, 利用局 部腐蚀法和台阶测厚仪测得薄膜厚度在 1.6 $\sim 2.0 \mu \mathrm{m}$ 之间. 样品的极化过程在两块极 板之间进行, 极板间距为 $2 \mathrm{~cm}$, 极化时两极 板之间所加电压为 $5000 \mathrm{~V}$, 温度为 $100^{\circ} \mathrm{C}$ (在硅油中). 二次谐波的产生采用一台 YAG: Nd 作为激发源, 将波长为 $1064 \mathrm{~nm}$ 的 平面偏振光从 $45^{\circ}$ 角的方向辐射薄膜表面, 用光电倍增管接收样品中产生的透射倍频光 信号.

在同样条件下对几类样品进行了二次谐 波强度的测试: 极化和未极化的 $\mathrm{BaTiO}_{3}$ 薄膜 和具有不同 $\mathrm{Ag}$ 纳米相体积分数 (以初始溶 液中 $\mathrm{Ag}$ 的摩尔比计算, $\mathrm{Ag}: \mathrm{Ba}=0.005$, $0.01,0.02)$, 样品的厚度基本相同或相近. 测试结果表明, 未经极化的样品所测得的二
次谐波信号很弱, 且与样品组分无关. 对于 刚刚极化后的样品, 则发现几种放埋 $\mathrm{Ag}$ 颗 粒的样品中产生的二次谐波强度均高于纯 $\mathrm{BaTiO}_{3}$ 样品, 其中 $\mathrm{Ag} / \mathrm{Ba}$ 值为 0.01 的样品 信号强度最高, 约为纯 $\mathrm{BaTiO}_{3}$ 样品的 4.6 倍, $\mathrm{Ag} / \mathrm{Ba}$ 值为 0.005 和 0.02 的样品的二次 谐波强度则分别是纯 $\mathrm{BaTiO}_{3}$ 样品的 2.1 和 2.8 倍. 根据二阶非线性光学系数与二次谐 波强度及样品厚度的关系 $\chi^{(2)} \propto \sqrt{I} / L(L$ 为 样品厚度), 从实验结果推算出 3 种嵌埋 $\mathrm{Ag}$ 颗粒的 $\mathrm{BaTiO}_{3}$ 薄膜样品的 $\chi^{(2)}$ 值分别相当 于纯 $\mathrm{BaTiO}_{3}$ 薄膜的 $1.4,1.9$ 和 1.6 倍. 由 此可见 $\mathrm{Ag}$ 纳米颗粒对 $\mathrm{BaTiO}_{3}$ 薄膜的二阶 非线性光学效应具有一定的增强作用.

族埋 $\mathrm{Ag}$ 纳米颗粒对极化的 $\mathrm{BaTiO}_{3}$ 薄 膜的二阶非线性光学效应的增强作用的机制 尚不十分清楚. 深入的研究工作尚在进行之 中.

\section{参考文献}

$1 \mathrm{Kim} \mathrm{D} \mathrm{H,} \mathrm{Kwok} \mathrm{H} \mathrm{S.} \mathrm{Plused} \mathrm{laser} \mathrm{deposition} \mathrm{of} \mathrm{BaTiO}_{3}$ thin films and their optical properties. Appl Phys Lett, 1995, 67(13): 1803

2 Zhou Ji, Li Longtu, Gui Zhilun, et al. Preparation and characterization of composites of ferroelectrics and nanoscale metal particals. Chinese Science Bulletin, 1995, 40(23): 2017

周 济『 李龙土 桂治轮『张孝文『 K.M.Moulding (2) D.J.Barber ${ }^{3}$ (1)清华大学材料科学与工程系, 北京 100084 ; (2)香港科技大学物理系, 香港) 